\title{
Relación entre la medición sonográfica de los músculos recto femoral y vasto intermedio y los parámetros bioquímicos convencionales para valorar el estado nutricional en la unidad de cuidados intensivos"
}

\author{
Alma Erika Hernández Plata, ${ }^{\star}$ Ma. Natalia Gómez González, ${ }^{*}$ Raúl Soriano Orozco, ${ }^{*}$ \\ Martha Alicia Hernández, ${ }^{*}$ Pedro Luis González Carrillo*
}

\section{RESUMEN}

Introducción: La desnutrición en el paciente crítico se relaciona con mal pronóstico. Las pérdidas proteicas se identifican de manera convencional mediante parámetros bioquímicos. La masa muscular puede reflejar la reserva corporal de proteínas, su medición por medio de sonografía ha evidenciado una fuerte correlación para detección de alteraciones.

Objetivo: Determinar la correlación de la medición sonográfica de los músculos Recto Femoral (RF) y Vasto Intermedio (VI), con los parámetros bioquímicos convencionales de valoración nutricional.

Métodos: Se estudiaron 12 pacientes mayores de 18 años, que recibieron dieta enteral temprana. Se midieron los parámetros bioquímicos, simultáneamente con parámetros sonográficos a nivel de RF y VI en los días uno y siete. Se estableció correlación entre los parámetros bioquímicos y sonográficos significativos.

Resultados: El parámetro bioquímico con mayor significancia estadística fue el Balance Nitrogenado Urinario (BNU) en muestra de orina de 24 horas $(\mathrm{p}=0.001)$. En mediciones sonográficas, el porcentaje de reducción muscular para RF y VI mostró mayor diferencia $(p=0.001)$. En análisis de correlación, se obtuvo fuerte correlación entre BNU y RF $(r=0.77)$ así como BNU y VI $(r=0.76)$.

Conclusión: Existe correlación entre las pérdidas proteicas medidas por BNU y el porcentaje de reducción de los músculos RF y VI medidos por sonografía. Palabras clave: Sonografía muscular, parámetros bioquímicos de valoración nutricional.

\section{SUMMARY}

Introduction: Malnutrition in critically ill patients is associated with poor prognosis. Protein losses are identified conventionally by biochemical parameters. The muscle mass can reflect the body protein reserve, its measurement by means of sonography has shown a strong correlation for the detection of alterations.

Objective: To determine the correlation of the sonographic measurement of the Rectus Femoris (RF) and Vastus Intermedius (VI) muscles, with the conventional biochemical parameters of nutritional assessment.

Methods: We studied 12 patients older than 18 years who received an early enteral diet. The biochemical parameters were measured, simultaneously with sonographic parameters at the level of RF and VI on days one and seven. Correlation was established between the significant biochemical and sonographic parameters.

Results: The biochemical parameter with the greatest statistical significance was the Urinary Nitrogen Balance (UNB) in a 24-hour urine sample $(p=0.001)$. In sonographic measurements, the percentage of muscle reduction for $R F$ and VI showed greater difference $(p=0.001)$. In correlation analysis, strong correlation was obtained between UNB and RF $(r=0.77)$ as UNB and $\mathrm{VI}(r=$ $0.76)$.

Conclusion: There is a correlation between the protein losses measured by UNB and the percentage reduction of RF and VI muscles measured by sonography.

Key words: Muscle Sonography, biochemical parameters of nutritional assessment.

\section{RESUMO}

Introdução: A desnutrição em pacientes críticos está associada a um mau prognóstico. As perdas de proteína são identificadas convencionalmente por parâmetros bioquímicos. A massa muscular pode refletir a reserva protéica corporal, sua medida por meio da ultrassonografia tem mostrado uma forte correlação para a detecção de alterações.

\footnotetext{
" Ganador del Premio Académico «Mario Shapiro». Tercer lugar.
}

* Unidad Médica de Alta Especialidad, Centro Médico Nacional Bajío Núm. 1. Instituto Mexicano del Seguro Social. León, Guanajuato, México.

Recepción: 30/08/2018. Aceptación: 17/11/2018.

Este artículo puede ser consultado en versión completa en http://www.medigraphic.com/medicinacritica
Objetivo: Determinar a correlação da medida ultrassonográfica dos músculos Reto Femoral (RF) e Vasto Intermediário (VI), com os parâmetros bioquímicos convencionais de avaliação nutricional.

Métodos: Foram estudados 12 pacientes com mais de 18 anos que receberam dieta enteral precocemente. Os parâmetros bioquímicos foram mensurados simultaneamente com parâmetros ultrassonográficos ao nível de FR e VI nos dias um e sete. Foi estabelecida correlação entre os parâmetros bioquímicos e ultrassonográficos significativos.

Resultados: $O$ parâmetro bioquímico com maior significância estatística foi o Balanço de Nitrogênio Urinário (NBU) em uma amostra de urina de 24 horas ( $p$ $=0,001$ ). Nas medidas ultrassonográficas, a porcentagem de redução muscular para FR e VI mostrou maior diferença $(p=0,001)$. Na análise de correlação, obteve-se forte correlação entre o BNU e FR $(r=0,77)$ e NBU e VI $(r=0,76)$. Conclusão: Existe uma correlação entre as perdas protéicas medidas pelo NBU e a redução percentual dos músculos $R F$ e VI medida pela ultra-sonografia. Palavras-chave: Sonografia muscular, parâmetros bioquímicos das avaliação nutricional.

\section{INTRODUCCIÓN}

La desnutrición continúa siendo la causa más frecuente del aumento de la morbimortalidad; afecta de manera exponencial particularmente a los pacientes hospitalizados. Es de peculiar interés el paciente crítico, en quien la incapacidad de ingesta y la enfermedad grave son comunes, tomando entidad propia bajo la denominación de «desnutrición hospitalaria» o «malnutrición». La desnutrición hospitalaria afecta a $30-50 \%$ de los pacientes hospitalizados de todas las edades, tanto por causas quirúrgicas como médicas, aumentando a medida que se prolonga la hospitalización. ${ }^{1}$ Así mismo, conforme avanza la desnutrición en el enfermo, se incrementa la morbilidad y mortalidad, siendo un factor importante para el pronóstico vital y funcional. La desnutrición hospitalaria del paciente crítico se presenta por dos condiciones importantes: la primera es por incapacidad del paciente para satisfacer sus necesidades y la segunda es por la respuesta metabólica frente a la lesión o enfermedad específica. $^{2}$

Los pacientes con enfermedad crítica muestran una respuesta hipercatabólica que se caracteriza por un incremento del gasto calórico secundario a la movilización de los carbohidratos, depósitos de lípidos y el catabolismo de las proteínas, que resulta en una pérdida de masa corporal magra. Dicha respuesta tiene la finalidad de asegurar niveles suficientes de sustratos circulantes en ausencia de ingesta, y es esta misma respuesta la que conduce a la aparición de una serie de trastornos que incrementan la morbilidad infecciosa, disfunción orgánica múltiple, hospitalización prolongada $y$, finalmente, la mortalidad en el paciente crítico, dependiendo de su duración e intensidad. ${ }^{2,3}$ Lo anterior 
resulta en una rápida disminución de la masa corporal magra que excede la asociada a reposo en cama o a una simple inanición. En el paciente crítico, la mayor pérdida muscular ocurre, por lo general, en los primeros 10 días posteriores a su ingreso a la unidad de cuidados intensivos (UCI), y estas pérdidas se incrementan por la severidad de la enfermedad. ${ }^{4}$ Además de ser un factor determinante para la morbimortalidad en la $\mathrm{UCI}$, se ha sugerido que el desgaste muscular durante una enfermedad crítica contribuye a la discapacidad funcional del sobreviviente. ${ }^{5}$ Adicionalmente, las reservas de masa muscular pueden reflejar el estado nutricional y reservas corporales de proteínas. ${ }^{6}$

Las características de los indicadores para la evaluación del estado nutricio deben ser: a) modificables a la intervención nutricia, b) confiables, c) reproducibles y d) susceptibles de validación. Los objetivos de la valoración de estado de nutrición son: a) conocer o estimar el estado de nutrición de un individuo o población en un momento dado, b) medir el impacto de la nutrición sobre la salud, el rendimiento o la supervivencia, c) identificar individuos en riesgo; prevenir la mala nutrición aplicando acciones profilácticas, planeación e implementación del manejo nutricio, d) monitorear, vigilar y confirmar la utilidad y validez clínica de los indicadores. ${ }^{7}$

El fallo en la detección y empeoramiento de la desnutrición en la estancia hospitalaria y en unidades de cuidados intensivos es muy común por el poco enfoque que se da a este rubro en comparación con otras medidas instauradas para el cuidado del paciente crítico. ${ }^{7}$ Para el diagnóstico del estado nutricional, se han estudiado y validado (con diferentes niveles de evidencia) algunos métodos bioquímicos, antropométricos e inmunológicos, todos ellos con sus ventajas y desventajas en su uso. En un reciente metaanálisis publicado por la Sociedad Americana de Nutrición Enteral y Parenteral (ASPEN, por sus siglas en inglés), se observó que los estudios publicados en los últimos cinco años para la evaluación del estado nutricional del paciente crítico continúan siendo parámetros convencionales; el indicador nutricional más comúnmente usado por este grupo es el peso corporal (53\%), seguido por parámetros bioquímicos (50\%), que incluyen prealbúmina, albúmina, hemoglobina y el balance nitrogenado. La literatura, sin embargo, ofrece una variedad de otras medidas que pueden considerarse para el uso diario en el entorno de la UCI. En forma ideal, tales medidas indicarían cómo los pacientes responden a la nutrición a corto plazo y se correlacionarían con los resultados a largo plazo de los pacientes. ${ }^{8}$

Los parámetros bioquímicos para la monitorización del estado nutricional del paciente crítico, según lo descrito por Ferrie y sus colaboradores (2017), pertenecen al grupo de estrategias de alta disponibilidad y moderada factibilidad; sin embargo, para la gran ma- yoría de estos parámetros, la confiabilidad es limitada. En el caso específico del balance nitrogenado urinario existen dificultades prácticas para garantizar una colección completa; es difícil de interpretar en pacientes con lesión renal aguda y existe un retraso de hasta 60 horas, antes de que un cambio en la ingesta se vea reflejado en la mejora de los resultados bioquímicos de control. ${ }^{9}$ Puede haber varias razones para estas limitaciones, como las alteraciones del estado hídrico, que pueden afectar las concentraciones séricas de varios de los indicadores bioquímicos más utilizados con más frecuencia. Así mismo, la respuesta de fase aguda de la enfermedad afecta a las proteínas viscerales o hepáticas, como la albúmina y prealbúmina, con independencia del estado nutricional o la información nutricional. ${ }^{10}$

Sin embargo, algunos estudios sugieren que si la respuesta de fase aguda es razonablemente estable, los niveles de prealbúmina se correlacionan con la ingesta de nutrición, ${ }^{11}$ pero quizás no con el resultado. La prealbúmina no está disponible en todos los laboratorios hospitalarios. El nivel de albúmina sérica se mide de forma rutinaria en la mayoría de los hospitales y es un fuerte indicador pronóstico incluso en enfermedades críticas, ${ }^{12}$ pero tiene una vida media larga y no responde con prontitud a una alteración significativa en el aporte nutricional. De manera similar, el recuento total de linfocitos está ampliamente disponible y es valioso como indicador de pronóstico en el seguimiento de la respuesta del paciente al soporte nutricional. ${ }^{13}$ Algunas pruebas de laboratorio menos comunes mencionadas en la literatura pueden ser mejores indicadores de nutrición que las pruebas de rutina antes descritas, entre ellas, el factor 1 de crecimiento similar a la insulina (ILGF-1) - también conocido como somatomedina-C-, que es una prueba menos común, pero parece viable como un indicador del balance de nitrógeno. Otras pruebas aún menos convencionales para evaluar el estado de la proteína del cuerpo incluyen los niveles en sangre $u$ orina de aminoácidos particulares o sus metabolitos..$^{8,14}$ Entre estos aminoácidos se encuentra la arginina, la cual aumenta en un estado catabólico. ${ }^{15}$ La tirosina no es sintetizada o degradada por el músculo; por lo tanto, la producción neta de tirosina debe representar una desintegración neta de la proteína. Los niveles de sangre de aminoácidos esenciales, aminoácidos condicionalmente esenciales ( $p$. ej. glutamina) o 3-metilhistidina urinaria (una medida de la degradación de proteínas, ya que no hay oxidación ni recaptación muscular de histidina) podrían ayudar a evaluar el estado de la proteína del paciente. Sin embargo, realizar un perfil completo de aminoácidos para cada paciente en la UCI puede no ser un método factible de control nutricional. ${ }^{16}$ Los cambios en la producción de aminoácidos pueden ocurrir en el músculo, pero no estar reflejados en los niveles plasmáticos; además, los perfiles de ami- 
noácidos pueden alterarse por otros factores, como por ejemplo, el uso de nutrición parenteral (ya que evita el metabolismo intestinal), lo cual dificulta la interpretación de los resultados. Así mismo, la creatinina sérica se ha sugerido como un marcador de masa muscular útil y disponible con facilidad. ${ }^{17,18}$

El músculo esquelético es fundamental para la función inmune y de citocinas; además, participa en cerca del $75 \%$ en la eliminación de glucosa. Por lo tanto, se espera que el desgaste muscular asociado a la $\mathrm{UCI}$ por múltiples factores como la inanición, postración y los propios del estado inflamatorio por la enfermedad de base y los cambios perjudiciales en la integridad muscular compliquen la regulación de la glucosa, los procesos inmunitarios e inflmatorios. Ante la respuesta sistémica a la lesión, existe una destrucción masiva de la masa muscular que se manifiesta por el aumento de las pérdidas urinarias de nitrógeno, que en los pacientes en estado crítico, son muy elevadas, traduciéndose en pérdida de masa muscular importante por día. ${ }^{16,19}$ Mantener y restaurar el músculo esquelético se convierte en un reto, dado que los pacientes exhiben un círculo vicioso de síntesis atenuada de proteína y aceleración de la descomposición de proteínas. Este círculo vicioso está impulsado por el reposo en cama relacionado con la $\mathrm{UCI}$, la ingesta calórica y proteica reducida, un estado proinflmatorio y resistencia anabólica e insulínica (definida como la capacidad reducida de absorber aminoácidos y glucosa en el músculo, respectivamente).5,6

Un indicador, hasta cierto punto novedoso y prometedor, es la medición del grosor del músculo o del área de la sección transversal mediante sonografía muscular. Ésta es una medida instantánea y no invasiva, pese a que se requiere un pequeño equipo de sonografía bidimensional para la medición; en la actualidad, su uso se ha hecho extensivo en las unidades de cuidados intensivos. En general, los estudios existentes se han centrado en el área de la sección transversal del músculo. ${ }^{20} \mathrm{El}$ ultrasonido ha demostrado sensibilidad de hasta $81 \%$ y una especificidad del $96 \%$ para la detección de cualquier alteración anormal del tejido muscular. ${ }^{20}$ Asimismo, múltiples estudios apoyan que las mediciones de ultrasonido de las longitudes de los fascículos musculares son confiables en un amplio rango de condiciones experimentales (coeficiente de correlación >0.6). La confiabilidad de las medidas de ecogenicidad es muy similar (coeficiente de correlación > 0.5). ${ }^{21}$

Las características del músculo esquelético pueden correlacionarse con el estado proteico del enfermo y proporcionar un enfoque más factible y objetivo para evaluar la salud muscular en pacientes de la UCl. Con el enfoque final en la actividad física y los resultados funcionales, no es sorprendente que los músculos cuádriceps, que son un importante grupo de músculos que soportan peso, hayan sido la región del cuerpo más estudiada. Se ha demostrado en forma repetida que la masa y la fuerza de los miembros inferiores se correlacionan en diversas poblaciones, incluso en los supervivientes. Aunque la masa muscular puede ser técnicamente una medida sustitutiva de la función muscular, no todos los estudios respaldan esta relación. Las cuantificaciones objetivas y subjetivas de los músculos (que incluyen, pero no se limitan, a la masa muscular, el grosor y el área de la sección transversal, medición de ecogenicidad) que son lo suficientemente sensibles para detectar pequeños cambios durante periodos agudos pueden, en última instancia, facilitar la evaluación de intervenciones para contrarrestar la atrofia y debilidad muscular. Durante la última década, las tecnologías de imágenes comunes como el ultrasonido y la tomografía computarizada (TC) se han introducido a la UCI para la cuantificación del músculo. La aplicación transformacional de estas técnicas ha avanzado la comprensión de las características musculares de los pacientes críticos en el momento del ingreso y durante la trayectoria de su estancia. ${ }^{22}$

La información sobre la composición muscular se puede recopilar mediante la cuantificación de la ecogenicidad muscular. Pillen y su grupo encontraron una correlación significativa entre la ecogenicidad y el tejido fibroso intersticial logrado a partir de biopsias musculares de perros. Además, se ha demostrado que los procesos de atrofia se correlacionan con una mayor acumulación de grasa intramuscular y tejido fibroso, que da como resultado una mayor ecogenicidad de ultrasonido. ${ }^{23}$ Este parámetro cualitativo-ecogenicidad se expresa de acuerdo con la escala de Heckmatt. El aumento de la ecogenicidad, en general, se considera como un índice de agotamiento de miofibrillas (Tabla 1). ${ }^{24}$

En cuanto a la disminución del área de sección transversal muscular, se estima que el $63 \%$ de los pacientes críticos tiene un área de sección transversal muscular baja medida por tomografía computarizada al momento de la admisión en la $\mathrm{UCl}$, y esta prevalencia aumenta a aproximadamente $70 \%$ cuando los pacientes tienen 65 años o más. La sonografía muscular ha demostrado alrededor de ocho a $30 \%$ de pérdida muscular en los primeros siete a 10 días de ingreso en la UCl. La disminución del AST puede traducir debilidad muscular; ésta, a su

Tabla 1: Escala de Heckmatt: escala de calificación visual para clasificar la intensidad del eco muscular. (Heckmatt JZ. Muscle nerve. 1988).

\begin{tabular}{cl}
\hline Grado & Apariencia sonográfica \\
\hline Grado 1 & Normal \\
Grado 2 & Aumento de la intensidad del eco muscular con eco óseo distintivo \\
Grado 3 & $\begin{array}{l}\text { Marcado aumento de la intensidad del eco muscular con un eco } \\
\text { óseo reducido }\end{array}$ \\
Grado 4 & Eco muscular muy fuerte y pérdida completa del eco óseo \\
\hline
\end{tabular}


vez, puede predecir de forma independiente los resultados clínicos, incluida la mortalidad, el tiempo dependiente del ventilador y la duración de la hospitalización. La atrofia muscular y la debilidad están quizá relacionadas con procesos catabólicos, como la inflamación durante y después de una enfermedad crítica, así como la inmovilidad o la falta de actividad. ${ }^{25,26}$ En un estudio realizado por Giuseppina y sus colegas, se observó una reducción significativa del músculo recto femoral a nivel del diámetro AP en todos los pacientes internados en la $\mathrm{UCl}$, que inició desde el día cinco. El diámetro LL no mostró disminución progresiva significativa, pero la diferencia entre el día cero y el día 20 fue significativa. EI AST dejó ver una reducción general del $45 \%$ los primeros 20 días, asociando estos cambios con mayor estancia en unidades de cuidados intensivos. ${ }^{27}$ La cuantificación precisa de los músculos es valiosa para caracterizar cambios metabólicos y funcionales en el tejido magro, para una comprensión de los mecanismos subyacentes del desgaste muscular y para la evaluación del éxito o fracaso de las intervenciones terapéuticas. Las adaptaciones metabólicas adversas secundarias a la atrofia muscular también pueden contribuir a estos pobres resultados funcionales y clínicos, pero la medición muscular es cada vez más fundamental en pacientes y supervivientes críticamente enfermos. Las medidas de composición corporal muscular, en específico por sonografía, presentan la oportunidad potencial de caracterizar de forma no invasiva la salud muscular midiendo el cambio en la cantidad y la calidad muscular.

La evaluación de los músculos esqueléticos por medio de la sonografía es no invasiva, simple, y puede repetirse cuantas veces sea necesario. Mediante este método podemos cuantificar los cambios morfológicos del músculo esquelético en pacientes críticos y relacionarlos con muchos parámetros, entre ellos, el nutricio. Una vez obtenidas las mediciones y habiéndose concluido un daño existente a nivel muscular, podemos basarnos en esta técnica para evaluar el impacto de diferentes estrategias terapéuticas sobre el desgaste muscular y nutricional. ${ }^{28}$ Por lo anterior, y ante el valor pronóstico que significa la medición de masa muscular en el paciente crítico, es importante contar de manera fiable con métodos de evaluación para detectar riesgos nutricionales. Aquellos pacientes con alto riesgo de desnutrición son los que se beneficiarán de una terapia nutricional temprana y agresiva.

\section{MATERIAL Y MÉTODOS}

Estudio longitudinal, prospectivo, observacional, realizado en una unidad de cuidados intensivos polivalente en el periodo comprendido entre diciembre de 2017 y marzo de 2018. Se ingresaron 12 pacientes mayores de 18 años con diagnósticos médicos, traumáticos y quirúrgicos, que recibieron dieta enteral en las primeras 24 horas posteriores a su ingreso, con aporte mínimo proteico de $0.8 \mathrm{mg} / \mathrm{kg} / \mathrm{día}$. Se realizó una evaluación general, así como una historia clínica que incluía edad, género e historia médica previa. Se tomó una muestra de sangre venosa y se inició la recolección de orina de 24 horas para la medición de parámetros bioquímicos basales al ingreso (balance nitrogenado, prealbúmina, albúmina, biometría hemática, química sanguínea, pruebas de funcionamiento hepático). Se realizó, así mismo, en las primeras 24 horas posteriores a su ingreso, una sonografía en la musculatura anterior de ambos muslos en dos ocasiones a nivel de los músculos recto femoral y vasto intermedio. Se registraron los siguientes parámetros de cada músculo: porcentaje de reducción muscular, diámetro AP, LL, AST y ecogenicidad muscular. Estas medidas fueron tomadas de manera sistematizada y bajo las mismas condiciones en el día siete de estancia en la UCI. Se excluyeron los pacientes con cualquier patología que afectara el área muscular a evaluar o amputación de los miembros pélvicos. Así mismo, aquellos pacientes que no alcanzaran este objetivo en la ingesta proteica enteral por cualquier motivo (intolerancia gastrointestinal, contraindicación a la alimentación enteral o suspensiones forzadas repetidas de alimentación enteral debido a procedimientos quirúrgicos múltiples), según lo establecido por guías ASPEN, o que hubieran cambiado a nutrición parenteral. Se analizaron los resultados por medio de programa estadístico SPSS versión 21.

Las mediciones sonográficas fueron realizadas a través de un sistema de ultrasonido portátil SonoScape S2 Color Doppler, un transductor convexo de $3.5 \mathrm{MHz}$, así como un transductor lineal de 9-11 Mhz; fueron tomadas por un único médico asignado, previamente entrenado. Las mediciones se realizaron en ambos muslos, con dos sesiones consecutivas, y se hizo un promedio de ellas. Las mediciones se efectuaron colocando al paciente en posición supina con las piernas en extensión. Con el transductor lineal a 90 grados del muslo, se llevaron a cabo lecturas a nivel del punto medio de una línea trazada de la espina iliaca anterosuperior al borde superior externo de la rótula: a ese nivel se tomó medición del RF y del diámetro AP; al girar el transductor para cortes transversos, se obtuvieron mediciones del diámetro LL. Se delimitó toda la masa muscular observada y se calculó el AST con los diámetros LL y AP. La segunda medición para el VI, músculo encontrado por debajo del $\mathrm{RF}$ e inmediatamente por arriba del fémur (Figura 1), se obtuvo a nivel de la unión del tercio medio con el tercio inferior de una línea trazada de la espina iliaca anterosuperior al borde superior de la rótula, y se obtuvieron mismos diámetros y área de superficie. Para finalizar, se tomó una medición cualitativa en un corte transversal, donde se comparó la ecogenicidad del músculo y del fémur, obteniendo valores para la escala según lo propuesto por Heckmatt (Heckmatt JZ. Muscle nerve. 1988). 

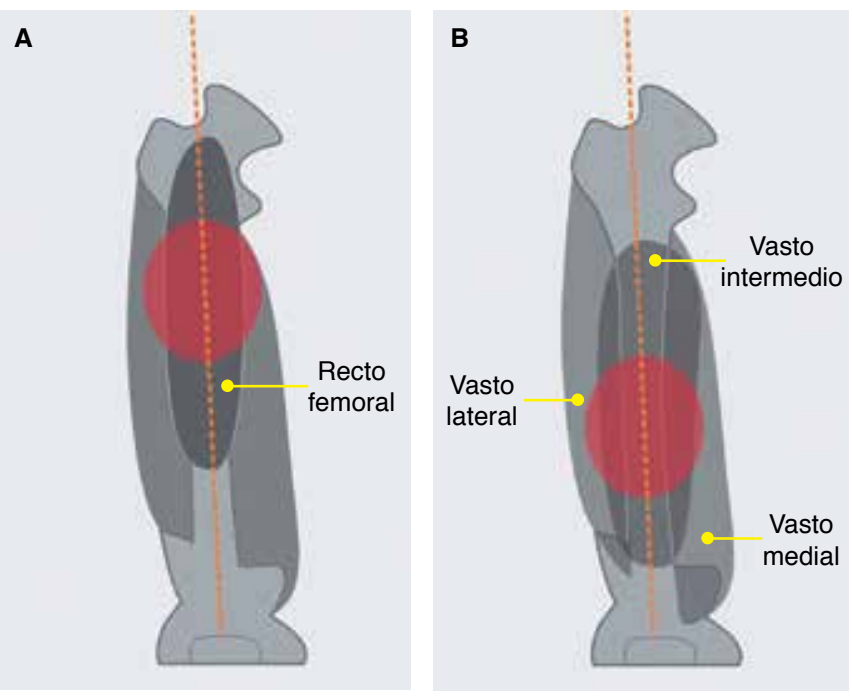

Figura 1: Anatomía muscular del muslo anterior. La primera medición es tomada en el músculo recto femoral (RF) en una línea imaginaria entre la espina iliaca anterosuperior y el borde superior de la rótula; se hacen mediciones sonográfias en el tercio medio (A). La segunda medición es tomada en el músculo vasto intermedio (VI) a nivel de la misma línea imaginaria, tercio inferior (B).

\section{RESULTADOS}

Se estudiaron 12 pacientes críticos, $62.5 \%$ hombres y $37.5 \%$ mujeres, con edad media de 36 años; $50 \%$ con IMC normal, $37.5 \%$ con sobrepeso y $12.5 \%$ con obesidad grado I. El aporte promedio de proteínas con dieta enteral temprana fue de $1.28 \mathrm{~g} / \mathrm{kg} /$ día y para el día siete de $1.84 \mathrm{~g} / \mathrm{kg} / \mathrm{día}$ ( $p=0.001)$, según los requerimientos guiados por el balance nitrogenado y las condiciones clínicas del paciente. No hubo intervención entre los resultados y las modificaciones realizadas en la nutrición por parte de los investigadores.

El diagnóstico más frecuente fue de origen traumático (62.5\%), seguido de médico en el 32\% (Tabla 2).

En el análisis de diferencia de medias a través de la prueba $t$, el parámetro bioquímico con diferencia más significativa entre los días uno y siete fue el balance nitrogenado urinario en muestra de orina de 24 horas, con una media de $3.1 \mathrm{~g} /$ día para el día uno y de $-4 \mathrm{~g} /$ día para el día siete $(p=0.001)$, seguido de la prealbúmina, con una media inicial de $22.7 \mathrm{mg} / \mathrm{L}$ y un descenso a 12 $\mathrm{mg} / \mathrm{L}$ en el día 7 ( $p=0.01$ ), y la albúmina, con medias de $3.04 \mathrm{mg} / \mathrm{dL}$ y $1.95 \mathrm{mg} / \mathrm{dL}$ para el día uno y siete, respectivamente, $(p=0.03)$. En el resto de los parámetros bioquímicos como leucocitos, hemoglobina, linfocitos y creatinina, no se encontró diferencia significativa.

En los parámetros sonográficos, los porcentajes de reducción muscular del RF y VI fueron los que presentaron mayor diferencia estadísticamente significativa entre ambos días de estudio, con medias de porcentaje de reducción de $17 \%$ para el RF y $16 \%$ para el VI ( $p=$
Tabla 2: Características bioquímicas y sonográficas del grupo de estudio en los días uno y siete. * Comparación de medias.

\begin{tabular}{|c|c|c|c|}
\hline Variable & Día uno & Día siete & $\mathrm{p}$ \\
\hline Edad (años) & 36 & -- & -- \\
\hline \multicolumn{4}{|l|}{ Sexo } \\
\hline a) Mujer & $37.5 \%$ & -- & -- \\
\hline b) Hombre & $62.5 \%$ & -- & -- \\
\hline \multicolumn{4}{|l|}{ Diagnóstico } \\
\hline a) Quirúrgico & $5.5 \%$ & -- & -- \\
\hline b) Médico & $32 \%$ & -- & -- \\
\hline c) Traumático & $62.5 \%$ & -- & -- \\
\hline Aporte proteico (g/kg/día) & 1.28 & 1.84 & 0.01 \\
\hline \multicolumn{4}{|l|}{ Parámetros bioquímicos } \\
\hline Leucocitos (x103/mL) & 15.7 & 13.8 & 0.41 \\
\hline Hemoglobina (g/dL) & 12.05 & 10.9 & 0.25 \\
\hline Linfocitos (\%) & 1.47 & 1.07 & 0.54 \\
\hline Creatinina $(\mathrm{mg} / \mathrm{dL})$ & 1.00 & 0.7 & 0.50 \\
\hline Albúmina $(\mathrm{g} / \mathrm{dL}))$ & 3.04 & 1.95 & 0.02 \\
\hline Prealbúmina (mg/dL) & 22.7 & 12 & 0.01 \\
\hline Balance nitrogenado ( $g / L)$ & 3.19 & -3.2 & 0.001 \\
\hline \multicolumn{4}{|l|}{ Parámetros sonográficos } \\
\hline Longitud AP del recto femoral (mm) & 13 & 10.3 & 0.08 \\
\hline Longitud LL del recto femoral (mm) & 37 & 30 & 0.02 \\
\hline $\begin{array}{l}\text { Área de sección transversal } \\
\text { del recto femoral }\left(\mathrm{cm}^{2}\right)\end{array}$ & 2.8 & 2.2 & 0.03 \\
\hline Porcentaje de reducción del recto femoral (\%) & 0 & -17.9 & 0.00 \\
\hline Longitud AP del vasto intermedio $(\mathrm{mm})$ & 16 & 1.2 & 0.04 \\
\hline Longitud LL del vasto intermedio (mm) & 35 & 28 & 0.08 \\
\hline $\begin{array}{l}\text { Área de sección transversal del vasto } \\
\text { intermedio }\left(\mathrm{cm}^{2}\right)\end{array}$ & 3.6 & 2.9 & 0.04 \\
\hline Porcentaje de reducción del vasto intermedio (\%) & -- & -16 & 0.00 \\
\hline Escala Heckmatt (grados) & 1.16 & 3.1 & 0.01 \\
\hline
\end{tabular}

*Prueba t de Student y equivalente no paramétrico: $\mathrm{U}$ de Mann-Whitney.

0.001 ), seguidos del AST del RF, con medias de 2.8 $\mathrm{cm}^{2}$ y $2.28 \mathrm{~cm}^{2}$ para los días uno y siete, respectivamente $(p=0.038)$, y AST del VI, con medias de $3.6 \mathrm{~cm}^{2}$ y $2.9 \mathrm{~cm}^{2}(p=0.04)$. En cuanto a las variables cualitativas de ecogenicidad muscular medidas por la escala de Heckmatt (Tabla 1), el 83\% de los pacientes ingresó al presente estudio en el grado 1 de ecogenicidad por dicha escala, y el $17 \%$ con grado 2 . Al final de la observación, el $9 \%$ permaneció con grado 2 , el $66 \%$ concluyó en grado 3 y el $25 \%$ en grado 4 , mostrando deterioro paulatino en la calidad de la miofibrilla.

Se realizó un análisis de correlación; el mayor valor se obtuvo entre el parámetro bioquímico de balance nitrogenado y los porcentajes de reducción para el RF y VI $(r=0.77$ y $r=0.76$, respectivamente); con ello, se otorgó una alta correlación entre estas dos variables (Figura 2). En cuanto a la albúmina, se observó una correlación moderada con el porcentaje de reducción del RF y VI, con una $r=0.51$ y $r=0.68$, respectivamente. Así mismo, la prealbúmina tuvo correlación moderada con el porcentaje de reducción del RF, con una $r=0.53$; no así con el porcentaje de reducción del $\mathrm{VI}$, donde se encontró una pobre correlación, con una $r=0.38$. La valoración de la ecogenicidad mediante la escala de Heckmatt también tuvo correlación de manera inversa 
con el balance nitrogenado $(r=-0.64)$. No se encontró relación entre las variables como hemoglobina, leucocitos, linfocitos, creatinina y los parámetros sonográficos medidos en este estudio.

La ingesta proteica tuvo impacto de manera inversa con los porcentajes de reducción para el músculo recto femoral $(r=-.579)$ y para el músculo vasto intermedio $(r$ $=-.535$ ) como resultado del catabolismo mostrado a lo largo de la estancia en la UCl en los primeros siete días (Tabla 3).

\section{DISCUSIÓN}

La desnutrición en el paciente crítico es un factor independiente de aumento de mortalidad, predisposición a enfermedades infecciosas y, por lo tanto, aumento en los días de estancia en la unidad de cuidados intensivos

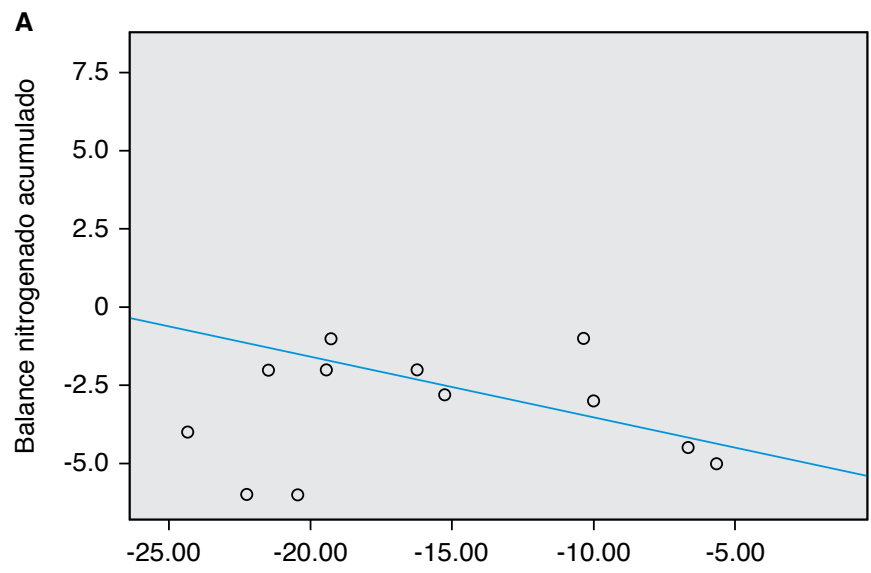

Porcentaje de reducción del recto femoral

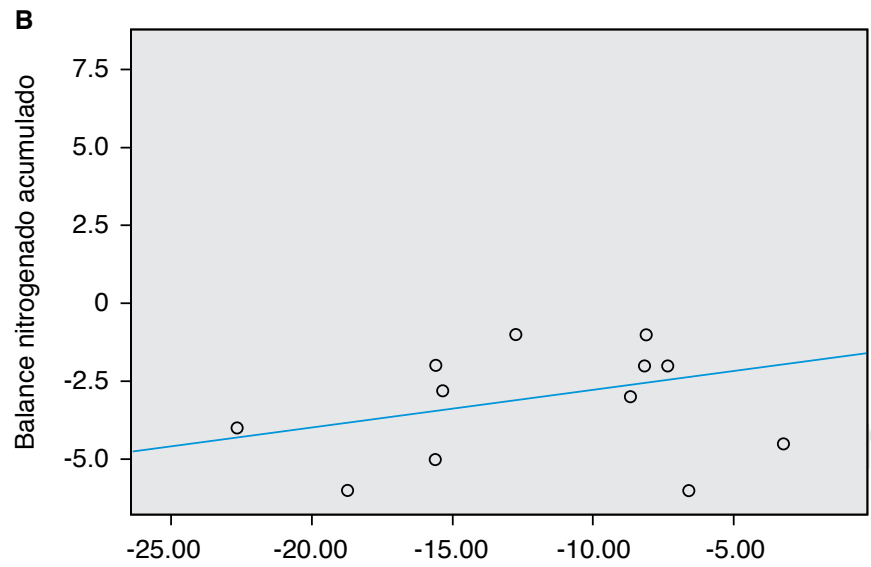

Porcentaje de reducción del músculo vasto intermedio

Figura 2: Gráfica de dispersión para la correlación entre el balance nitrogenado acumulado y el porcentaje de reducción del músculo recto femoral al séptimo día. En el análisis de correlación bivariada, una $r=0.77$ con valor de $p=0.001(\boldsymbol{A})$. Gráfica de dispersión para la correlación entre el balance nitrogenado acumulado y el porcentaje de reducción del músculo vasto intermedio al séptimo día. En el análisis de correlación bivariada, una $r=0.76$, con valor de $p=0.003$ (B). y ventilación mecánica. El retraso en el diagnóstico del estado nutricio en esta población impacta en un mayor deterioro orgánico, a pesar de todas las medidas óptimas instauradas. Si se realiza una detección temprana de la desnutrición en el paciente crítico, aun antes de los resultados de los parámetros bioquímicos convencionales de valoración del estado nutricional, podremos incidir de manera efectiva en un tratamiento nutricional óptimo y, de esa manera, mejorar el pronóstico vital y funcional en estos pacientes.

En el presente estudio investigamos los parámetros bioquímicos que comúnmente utilizamos en las unidades de terapia intensiva para valorar el estado nutricional y buscamos correlación con medidas sonográficas específicas y de fácil acceso. En este caso, usamos las medidas de los músculos anteriores del muslo, las cuales ya están validadas y aceptadas como confiables, como lo muestra el estudio de Giles y sus colaboradores (2015), con una correlación cercana al $r=0.91$; en dicho trabajo se sugiere que la medición del grosor muscular con este método se puede utilizar como un sustituto cuando la resonancia magnética no sea un recurso disponible. La sonografía muscular también se puede usar como una estimación conservadora de la atrofia selectiva o pérdida del porcentaje muscular.

El balance nitrogenado en orina de 24 horas ha sido una herramienta tradicional en la valoración nutricia;

Tabla 3: Correlación de las variables sonográficas y bioquímicas. ${ }^{*}$

\begin{tabular}{|c|c|c|c|}
\hline Variables & $\begin{array}{l}\text { Balance } \\
\text { nitrogenado }\end{array}$ & Prealbúmina & Albúmina \\
\hline Aporte proteico & -0.79 & 0.23 & -0.39 \\
\hline \multicolumn{4}{|l|}{ Parámetros bioquímicos } \\
\hline Leucocitos & 0.14 & 0.46 & 0.09 \\
\hline Hemoglobina & 0.28 & -0.07 & 0.65 \\
\hline Linfocitos & 0.21 & 0.13 & 0.5 \\
\hline Creatinina & 0.32 & 0.17 & 0.57 \\
\hline Albúmina & 0.43 & 0.19 & 1 \\
\hline Prealbúmina & 0.47 & 1 & 0.19 \\
\hline Balance nitrogenado & 1 & 0.47 & 0.43 \\
\hline \multicolumn{4}{|l|}{ Parámetros sonográficos } \\
\hline Longitud AP del recto femoral & 0.5 & 0.01 & 0.31 \\
\hline Longitud LL del recto femoral & 0.75 & 0.20 & 0.53 \\
\hline $\begin{array}{l}\text { Área de sección transversal del } \\
\text { recto femoral }\end{array}$ & 0.58 & 0.25 & 0.43 \\
\hline $\begin{array}{l}\text { Porcentaje de reducción del } \\
\text { recto femoral }\end{array}$ & 0.77 & 0.53 & 0.51 \\
\hline $\begin{array}{l}\text { Longitud AP del vasto } \\
\text { intermedio }\end{array}$ & 0.48 & 0.11 & 0.35 \\
\hline $\begin{array}{l}\text { Longitud LL del vasto } \\
\text { intermedio }\end{array}$ & 0.43 & 0.14 & 0.33 \\
\hline $\begin{array}{l}\text { Área de sección transversal del } \\
\text { vasto intermedio }\end{array}$ & 0.42 & 0.25 & 0.09 \\
\hline $\begin{array}{l}\text { Porcentaje de reducción del } \\
\text { vasto intermedio }\end{array}$ & 0.76 & 0.38 & 0.68 \\
\hline Escala Heckmatt & 0.82 & -0.34 & 0.77 \\
\hline
\end{tabular}

*Coeficiente de correlación producto-momento de Pearson y equivalente no paramétrico: correlación de Spearman. 
aunque es bien sabido que no en todos los pacientes se puede aplicar este parámetro como seguimiento nutricio por factores que impiden su adecuada recolección e interpretación, continúa su vigencia dentro de las estrategias de valoración y terapéutica nutricional del paciente crítico, ya que es una medición con alta disponibilidad y factibilidad en las terapias intensivas que se encuentra contemplado en las guías internacionales como ASPEN (Ferrie y su grupo, 2017).

En cuanto a los resultados del balance nitrogenado en 24 horas obtenidos en este estudio, observamos en primer lugar que nuestros pacientes se encontraban al ingreso a la terapia intensiva con balances nitrogenados con tendencia al anabolismo o balance neutro; sin embargo, a medida que transcurrieron los siete días de la segunda medición, notamos balances característicamente negativos en todos los pacientes, incluso en rangos de catabolismo severo. El tipo de paciente y la causa de la admisión en la UCI podrían estar correlacionados con dicho hipercatabolismo, ya que los pacientes en su gran mayoría fueron de origen traumático $(62 \%)$, mientras que aquéllos en cirugía electiva postoperatoria tuvieron un valor en el balance nitrogenado menos negativo. Todo esto se observó a pesar de que la ingesta proteica se optimizaba hasta alcanzar valores máximos, según lo recomendado en las guías internacionales.

Se notó que el balance nitrogenado en valores catabólicos moderados tiene correlación con la disminución del porcentaje del RF y VI $(r=0.66)$ y con la calidad de la miofibrilla medida por la escala cualitativa de Heckmatt ( $r=0.7)$, lo que que el deterioro muscular medido por sonografía se relaciona con la pérdida proteica observada en los balances nitrogenados urinarios.

En cuanto al resto de las variables bioquímicas, en el presente estudio vimos lo descrito por Ferrie y su grupo (2017) con respecto a la poca sensibilidad de otros biomarcadores como linfocitos, hemoglobina y creatinina, que no obtuvieron valores significativos entre la valoración inicial y la final.

Tener una correlación del desgaste muscular por medio de sonografía con los parámetros bioquímicos disponibles hasta la fecha podría ser de utilidad como marcador de diagnóstico temprano del estado nutricional del paciente crítico, lo que resultaría en una oportuna y eficaz modificación del aporte nutricional.

La evaluación secuencial de los cambios cuantitativos y cualitativos de la masa muscular puede ayudar a identificar a los pacientes críticamente enfermos con alto riesgo de desnutrición y disfunción muscular, así como a verificar los efectos de diferentes regímenes nutricionales. En este sentido, la evaluación sonográfica de los músculos esqueléticos (en particular, el recto femoral y el vasto intermedio) es una herramienta confiable para valorar los cambios musculares a lo largo del tiempo; es inocua, no invasiva y se realiza a la cabecera del paciente. Su costo-efectividad es más bajo que muchas otras técnicas como la tomografía computarizada, la resonancia magnética, etcétera, que han sido utilizadas con el mismo propósito. De esta forma, se puede optimizar el uso de recursos materiales y humanos para la atención del paciente crítico.

El presente estudio tiene varias limitaciones, algunas de las cuales son inherentes a su naturaleza observacional, en donde no fue posible influir en las estrategias terapéuticas, tanto clínicas como nutricionales. Otra limitación es la gran diversidad entre los tipos de pacientes en una terapia intensiva polivalente; factores como el estado nutricional previo y el tipo de nutrición derivan en diferencias en los resultados. Además, el número de pacientes puede no ser lo suficientemente grande como para responder preguntas sobre grupos específicos, como pacientes con traumatismo, con naturaleza quirúrgica o médica. Esto ofrece un área de oportunidad para subsecuentes trabajos donde se pueda aclarar mejor el panorama del balance nitrogenado, el cual sigue y seguirá vigente como método de valoración del estado nutricional del paciente crítico y su correlación con modernos métodos para ese fin, como la sonografía.

\section{CONCLUSIÓN}

El porcentaje de reducción muscular del músculo recto femoral y del vasto intermedio, así como el desgaste de las miofibrillas medido por escala cualitativa de Heckmatt, obtenidos por sonografía, tienen correlación con el balance nitrogenado, por lo que pueden ser parte del monitoreo nutricional para detectar de manera oportuna riesgo o desnutrición incipiente.

La ventaja sobre los parámetros nutricionales es que la medición sonográfica es dinámica y repetible cuantas veces sea necesario a la cabecera del paciente, sin necesidad de esperar 24 horas para un balance urinario; además, puede ser el monitoreo de elección en los pacientes con lesión renal aguda, los cuales, como se sabe, pueden cursar con oligo-anuria.

La utilidad de la sonografía muscular propuesta en este estudio no sólo se limita al monitoreo nutricional, sino que también tiene futuro como valoración en aquellos pacientes en los que se desea iniciar rehabilitación oportuna y funcional, disminuyendo el tiempo de postración y sus complicaciones conocidas.

\section{BIBLIOGRAFÍA}

1. Mourão F, Amado D, Ravasco P, Vidal PM, Camilo ME Nutritional risk and status assessment in surgical patients: a challenge amidst plenty. Nutr Hosp. 2004;19(2):83-88.

2. Costelli P, Baccino FM. Mechanisms of skeletal muscle depletion in wasting syndromes: role of ATP-ubiquitin-dependent proteolysis. Curr Opin Clin Nutr Metab Care. 2003;6(4):407-412. 
3. Mourtzakis M, Wischmeyer P. Bedside ultrasound measurement of skeletal muscle. Curr Opin Clin Nutr Metab Care. 2014;17(5):389-395.

4. McClave SA, Taylor BE, Martindale RG, Warren MM, Johnson $\mathrm{DR}$, Braunschweig $\mathrm{C}$, et al. Guidelines for the Provision and Assessment of Nutrition Support Therapy in the Adult Critically III Patient: Society of Critical Care Medicine (SCCM) and American Society for Parenteral and Enteral Nutrition (ASPEN.). JPEN J Parenter Enteral Nutr. 2016;40(2):159-211.

5. Puthucheary ZA, McNelly AS, Rawal J, Connolly B, Sidhu PS, Rowlerson A, et al. Rectus femoris cross-sectional area and muscle layer thickness: comparative markers of muscle wasting and weakness. Am J Respir Crit Care Med. 2017;195(1):136-138.

6. Costa-Moreira O, Patrocinio de Oliveira CE, Candia-Luján R, Romero-Pérez EM, de Paz-Fernández JA. Métodos de evaluación de la masa muscular: una revisión sistemática de ensayos controlados aleatorios. Nutr Hosp. 2015;32(3):977-985.

7. Montejo-González JC, Culebras-Fernández JM, García de Lorenzo y Mateos A. Recomendaciones para la valoración nutricional del paciente crítico. Rev Méd Chile. 2006;134(8):1049-1056.

8. Ferrie S, Tsang E. Monitoring nutrition in critical illness: what can we use? Nutr Clin Pract. 2017:884533617706312.

9. Ferrie S, Allman-Farinelli M. Commonly used "nutrition" indicators do not predict outcome in the critically ill: a systematic review. Nutr Clin Pract. 2013;28(4):463-484.

10. Hill GL, Witney GB, Christie PM, Church JM. Protein status and metabolic expenditure determine the response to intravenous nutrition - a new classification of surgical malnutrition. Br J Surg. 1991;78(1):109-113.

11. Casati A, Muttini S, Leggieri C, Colombo S, Giorgi E, Torri G. Rapid turnover proteins in critically ill ICU patients. Negative acute phase proteins or nutritional indicators? Minerva Anestesiol. 1998;64(7-8):345-350.

12. Apelgren KN, Rombeau JL, Twomey PL, Miller RA. Comparison of nutritional indices and outcome in critically ill patients. Crit Care Med. 1982;10(5):305-307.

13. Fletcher JP, Little JM, Walker PJ. The relationship of nutritional parameters to mortality and septic complications. Aust $N Z J$ Surg. 1986;56(12):891-895.

14. Rogers AJ, McGeachie M, Baron RM, Gazourian L, Haspel $\mathrm{JA}$, Nakahira K, et al. Metabolomic derangements are associated with mortality in critically ill adult patients. PLoS One. 2014;9(1):e87538.

15. Luiking YC, Poeze M, Ramsay G, Deutz NE. The role of arginine in infection and sepsis. JPEN J Parenter Enteral Nutr. 2005;29(1 Suppl):S70-S74.

16. Askanazi J, Carpentier YA, Michelsen CB, Elwyn DH, Furst $P$, Kantrowitz LR, et al. Muscle and plasma amino acids following injury. Influence of intercurrent infection. Ann Surg. 1980;192(1):78-85.

17. Ballesteros $\mathrm{M}$, Cortes A, Arleaga Cl, Puerto R, Bojac B. The usefulness of serum creatinine levels in identifying hospital malnutrition. Nutr Hosp. 1994;9(3):186-196.
18. Cheatham ML, Safcsak K, Brzezinski SJ, Lube MW. Nitrogen balance, protein loss, and the open abdomen. Crit Care Med. 2007;35(1):127-131.

19. Brockmann K, Becker P, Schreiber G, Neubert K, Brunner E, Bönnemann C. Sensitivity and specificity of qualitative muscle ultrasound in assessment of suspected neuromuscular disease in childhood. Neuromuscul Disord. 2007;17(7):517-523.

20. Pillen S, van Alfen N. Skeletal muscle ultrasound. Neurol Res. 2011;33(10):1016-1024.

21. Kwah LK, Pinto RZ, Diong J, Herbert RD. Reliability and validity of ultrasound measurements of muscle fascicle length and pennation in humans: a systematic review. J Appl Physiol (1985). 2013;114(6):761-769.

22. Heyland DK, Dhaliwal $R$, Jiang $X$, Day AG. Identifying critically ill patients who benefit the most from nutrition therapy: the development and initial validation of a novel risk assessment tool. Crit Care. 2011;15(6):R268.

23. Paris MT, Mourtzakis M, Day A, Leung R, Watharkar S, Kozar R, et al. Validation of bedside ultrasound of muscle layer thickness of the quadriceps in the critically ill patient (validum study). JPEN $J$ Parenter Enteral Nutr. 2017;41(2):171-180.

24. Pillen S, Tak RO, Zwarts MJ, Lammens MM, Verrijp KN, Arts $\mathrm{IM}$, et al. Skeletal muscle ultrasound: correlation between fibrous tissue and echo intensity. Ultrasound Med Biol. 2009;35(3):443446.

25. Grimm A, Teschner U, Porzelius C, Ludewig K, Zielske J, Witte OW, et al. Muscle ultrasound for early assessment of critical illness neuromyopathy in severe sepsis. Crit Care. 2013;17(5):R227.

26. Strasser EM, Draskovits T, Praschak M, Quittan M, Graf A Association between ultrasound measurements of muscle thickness, pennation angle, echogenicity and skeletal muscle strength in the elderly. Age (Dordr). 2013;35(6):2377-2388.

27. Annetta MG, Pittiruti M, Silvestri D, Grieco DL, Maccaglia A, La Torre MF, et al. Ultrasound assessment of rectus femoris and anterior tibialis muscles in young trauma patients. Ann Intensive Care. 2017;7(1):104.

28. Mourtzakis M, Parry S, Connolly B, Puthucheary Z. Skeletal muscle ultrasound in critical care: a tool in need of translation. Ann Am Thorac Soc. 2017;14(10):1495-1503.

Patrocinios y conflicto de intereses: Los autores declaramos no haber recibido patrocinio para el presente trabajo ni tener conflicto de intereses.

Correspondencia:

Dra. Alma Erika Hernández Plata

E-mail: kika_1980@hotmail.com 